\title{
Erratum: New genetic loci implicated in fasting glucose homeostasis and their impact on type 2 diabetes risk
}

Josée Dupuis, Claudia Langenberg, Inga Prokopenko, Richa Saxena, Nicole Soranzo, Anne U Jackson, Eleanor Wheeler, Nicole L Glazer, Nabila Bouatia-Naji, Anna L Gloyn, Cecilia M Lindgren, Reedik Mägi, Andrew P Morris, Joshua Randall, Toby Johnson, Paul Elliott, Denis Rybin, Gudmar Thorleifsson, Valgerdur Steinthorsdottir, Peter Henneman, Harald Grallert, Abbas Dehghan, Jouke Jan Hottenga, Christopher S Franklin, Pau Navarro, Kijoung Song, Anuj Goel, John R B Perry, Josephine M Egan, Taina Lajunen, Niels Grarup, Thomas Sparsø, Alex Doney, Benjamin F Voight, Heather M Stringham, Man Li, Stavroula Kanoni, Peter Shrader, Christine Cavalcanti-Proença, Meena Kumari, Lu Qi, Nicholas J Timpson, Christian Gieger, Carina Zabena, Ghislain Rocheleau, Erik Ingelsson, Ping An, Jeffrey O'Connell, Jian'an Luan, Amanda Elliott, Steven A McCarroll, Felicity Payne, Rosa Maria Roccasecca, François Pattou, Praveen Sethupathy, Kristin Ardlie, Yavuz Ariyurek, Beverley Balkau, Philip Barter, John P Beilby, Yoav Ben-Shlomo, Rafn Benediktsson, Amanda J Bennett, Sven Bergmann, Murielle Bochud, Eric Boerwinkle, Amélie Bonnefond, Lori L Bonnycastle, Knut Borch-Johnsen, Yvonne Böttcher, Eric Brunner, Suzannah J Bumpstead, Guillaume Charpentier, Yii-Der Ida Chen, Peter Chines, Robert Clarke, Lachlan J M Coin, Matthew N Cooper, Marilyn Cornelis, Gabe Crawford, Laura Crisponi, Ian N M Day, Eco J C de Geus, Jerome Delplanque, Christian Dina, Michael R Erdos, Annette C Fedson, Antje Fischer-Rosinsky, Nita G Forouhi, Caroline S Fox, Rune Frants, Maria Grazia Franzosi, Pilar Galan, Mark O Goodarzi, Jürgen Graessler, Christopher J Groves, Scott Grundy, Rhian Gwilliam, Ulf Gyllensten, Samy Hadjadj, Göran Hallmans, Naomi Hammond, Xijing Han, Anna-Liisa Hartikainen, Neelam Hassanali, Caroline Hayward, Simon C Heath, Serge Hercberg, Christian Herder, Andrew A Hicks, David R Hillman, Aroon D Hingorani, Albert Hofman, Jennie Hui, Joe Hung, Bo Isomaa, Paul R V Johnson, Torben Jørgensen, Antti Jula, Marika Kaakinen, Jaakko Kaprio, Y Antero Kesaniemi, Mika Kivimaki, Beatrice Knight, Seppo Koskinen, Peter Kovacs, Kirsten Ohm Kyvik, G Mark Lathrop, Debbie A Lawlor, Olivier Le Bacquer, Cécile Lecoeur, Yun Li, Valeriya Lyssenko, Robert Mahley, Massimo Mangino, Alisa K Manning, María Teresa Martínez-Larrad, Jarred B McAteer, Laura J McCulloch, Ruth McPherson, Christa Meisinger, David Melzer, David Meyre, Braxton D Mitchell, Mario A Morken, Sutapa Mukherjee, Silvia Naitza, Narisu Narisu, Matthew J Neville, Ben A Oostra, Marco Orrù, Ruth Pakyz, Colin N A Palmer, Giuseppe Paolisso, Cristian Pattaro, Daniel Pearson, John F Peden, Nancy L Pedersen, Markus Perola, Andreas F H Pfeiffer, Irene Pichler, Ozren Polasek, Danielle Posthuma, Simon C Potter, Anneli Pouta, Michael A Province, Bruce M Psaty, Wolfgang Rathmann, Nigel W Rayner, Kenneth Rice, Samuli Ripatti, Fernando Rivadeneira, Michael Roden, Olov Rolandsson, Annelli Sandbaek, Manjinder Sandhu, Serena Sanna, Avan Aihie Sayer, Paul Scheet, Laura J Scott, Udo Seedorf, Stephen J Sharp, Beverley Shields, Gunnar Sigurðsson, Eric J G Sijbrands, Angela Silveira, Laila Simpson, Andrew Singleton, Nicholas L Smith, Ulla Sovio, Amy Swift, Holly Syddall, Ann-Christine Syvänen, Toshiko Tanaka, Barbara Thorand, Jean Tichet, Anke Tönjes, Tiinamaija Tuomi, André G Uitterlinden, Ko Willems van Dijk, Mandy van Hoek, Dhiraj Varma, Sophie Visvikis-Siest, Veronique Vitart, Nicole Vogelzangs, Gérard Waeber, Peter J Wagner, Andrew Walley, G Bragi Walters, Kim L Ward, Hugh Watkins, Michael N Weedon, Sarah H Wild, Gonneke Willemsen, Jaqueline C M Witteman, John W G Yarnell, Eleftheria Zeggini, Diana Zelenika, Björn Zethelius, Guangju Zhai, Jing Hua Zhao, M Carola Zillikens, DIAGRAM Consortium, GIANT Consortium, Global BPgen Consortium, Ingrid B Borecki, Ruth J F Loos, Pierre Meneton, Patrik K E Magnusson, David M Nathan, Gordon H Williams, Andrew T Hattersley, Kaisa Silander, Veikko Salomaa, George Davey Smith, Stefan R Bornstein, Peter Schwarz, Joachim Spranger, Fredrik Karpe, Alan R Shuldiner, Cyrus Cooper, George V Dedoussis, Manuel Serrano-Ríos, Andrew D Morris, Lars Lind, Lyle J Palmer, Frank B Hu, Paul W Franks, Shah Ebrahim, Michael Marmot, W H Linda Kao, James S Pankow, Michael J Sampson, Johanna Kuusisto, Markku Laakso, Torben Hansen, Oluf Pedersen, Peter Paul Pramstaller, H Erich Wichmann, Thomas Illig, Igor Rudan, Alan F Wright, Michael Stumvoll, Harry Campbell, James F Wilson, Anders Hamsten on behalf of Procardis Consortium, Richard N Bergman, Thomas A Buchanan, Francis S Collins, Karen L Mohlke, Jaakko Tuomilehto, Timo T Valle, David Altshuler, Jerome I Rotter, David S Siscovick, Brenda W J H Penninx, Dorret I Boomsma, Panos Deloukas, Timothy D Spector, Timothy M Frayling, Luigi Ferrucci, Augustine Kong, Unnur Thorsteinsdottir, Kari Stefansson, Cornelia M van Duijn, Yurii S Aulchenko, Antonio Cao, Angelo Scuteri, David Schlessinger, Manuela Uda, Aimo Ruokonen, Marjo-Riitta Jarvelin, Dawn M Waterworth, Peter Vollenweider, Leena Peltonen, Vincent Mooser, Goncalo R Abecasis, Nicholas J Wareham, Robert Sladek, Philippe Froguel, Richard M Watanabe, James B Meigs, Leif Groop, Michael Boehnke, Mark I McCarthy, Jose C Florez \& Inês Barroso for the MAGIC investigators

Nat. Genet. 42, 105-116 (2010); published online 17 January 2010; corrected after print 26 March 2010

In the version of this article initially published, there were several errors in the author affiliations. These errors have been corrected in the HTML and PDF versions of the article.

\section{Corrigendum: A TARBP2 mutation in human cancer impairs microRNA processing and DICER1 function}

Sonia A Melo, Santiago Ropero, Catia Moutinho, Lauri A Aaltonen, Hiroyuki Yamamoto, George A Calin, Simona Rossi, Agustin F Fernandez, Fatima Carneiro, Carla Oliveira, Bibiana Ferreira, Chang-Gong Liu, Alberto Villanueva, Gabriel Capella, Simo Schwartz Jr, Ramin Shiekhattar \& Manel Esteller

Nat. Genet. 41, 365-370 (2009); published online 15 February 2009; corrected after print 9 April 2010

In the version of this article initially published, the colony formation assay image labeled "Co115.DICER1" was the incorrect image. The error has been corrected and a corrected version of Figure 4 panel e is provided in the HTML and PDF versions of the article. 